\title{
An Orthogonalized Valence Orbital Approximation in Relativistic Full-Potential Linear-Combination-of-Atomic-Orbitals Methods
}

\author{
Shugo Suzuki and Toshihiro ArIIZumi \\ Institute of Materials Science, University of Tsukuba, Tsukuba 305-8573
}

(Received December 6, 2006)

An approximation for reducing the computational cost in fully relativistic and scalar relativistic full-potential linear-combination-of-atomic-orbitals (LCAO) methods is introduced. The basic idea is the same as in the orthogonalized plane wave method; valence orbitals are orthogonalized to core orbitals and, using the orthogonalized valence orbitals as the basis functions in the generalized eigenvalue problem, the size of matrices is reduced considerably for materials with heavy atoms. This enables us to calculate their structural and electronic properties efficiently. The usefulness of the orthogonalized valence orbital approximation is shown by applying it to $\mathrm{Au}, \mathrm{InSb}$, and AmBi. In particular, it is found that the effect of spin-orbit coupling is reproduced successfully in the fully relativistic full-potential LCAO calculations within the orthogonalized valence orbital approximation.

\section{KEYWORDS: orthogonalized valence orbitals, fully relativistic calculations, scalar relativistic calculations, full-potential calculations, LCAO method, density functional theory, band calculations, structure optimizations}

\section{Introduction}

The first-principles study of the structural and electronic properties of materials with heavy atoms on the basis of density functional theory has been developed extensively in the past two decades. ${ }^{1,2)}$ Several methods of calculations are now commonly employed: the augmented-plane-wave method, ${ }^{3-8)}$ the Korringa-Kohn-Rostoker Green's function method, ${ }^{9-16)}$ the linear-muffin-tin-orbital method, ${ }^{17-22)}$ and the linear-combination-of-atomicorbitals (LCAO) method. ${ }^{23-29)}$ In these methods, two important features are indispensable for realistic calculations; one is that they utilize the full-potential approach where the electrostatic potential is calculated accurately without any kinds of shape approximation and the other is that they deal with relativistic effects adequately according to the accuracy required.

So far, there have been extensive studies utilizing first-principles fully relativistic calculations in the sense that the Dirac-Kohn-Sham equations are solved directly. ${ }^{30)}$ Although the perturbation approach to spin-orbit coupling is sufficiently good for materials with light atoms, the fully relativistic approach is still desirable for materials with heavy atoms when the spin-orbit coupling should be taken into account accurately without approximations such as the expansion in $1 / c^{2}$, where $\mathrm{c}$ is the speed of light. A few studies, however, have been reported 
which employ not only fully relativistic calculations but also full-potential calculations. ${ }^{16,28)}$ In our previous paper, ${ }^{28)}$ we have shown that a fully relativistic full-potential LCAO (FFLCAO) method is useful for studying the structural and electronic properties of materials with heavy atoms. Unfortunately, the computational cost of the FFLCAO method is high in comparison with that of the nonrelativistic LCAO method; the FFLCAO method uses two times more basis functions and thus involves calculating four times more matrix elements. Furthermore, the basis functions themselves are four-component spinors. As a result, the computational cost of the FFLCAO method is more than ten times higher than that of the nonrelativistic LCAO method. This difficulty is serious especially for materials with heavy atoms because the number of basis functions is very large for heavy atoms. One way to reduce the computational cost is to adopt the scalar relativistic full-potential LCAO (SFLCAO) method where all the relativistic effects except the spin-orbit coupling are incorporated. ${ }^{29)}$ Nevertheless, a fully relativistic approach is still desirable when one needs to study the properties where accurate calculations of the spin-orbit coupling are necessary.

A point to be noted when the FFLCAO method is applied to materials with heavy atoms is that the core orbitals are handled in the same manner as the valence orbitals, although the number of core orbitals is very large. The computational cost is thus expected to be reduced considerably if we deal with the core orbitals in a simplified manner as implemented in the other methods. This can be achieved by orthogonalizing the valence orbitals to the core orbitals and then by eliminating the core orbitals from the generalized eigenvalue problem; the basic idea was originally proposed in 1940 as the orthogonalized plane wave method ${ }^{31)}$ and later it was extended as the orthogonalized LCAO method. ${ }^{32)}$ It is thus expected that the use of orthogonalized valence orbitals (OVO) in the FFLCAO method can also reduce the computational cost considerably.

The purpose of the present paper is to show the usefulness of the OVO approximation (OVOA) in the FFLCAO method as well as in the SFLCAO method. In $\S 2$, the OVOA is described in detail. We show in $\S 3$ that the OVOA is useful for studying the structural and electronic properties of materials with heavy atoms by applying it to $\mathrm{Au}$ and InSb. Furthermore, in $\S 4$, the electronic structure of $\mathrm{AmBi}$ is studied within and without the OVOA to show the applicability of the present method to materials with very heavy atoms. Finally, conclusions are given in $\S 5$.

\section{Orthogonalized Valence Orbital Approximation}

We now describe the OVOA in detail. The first step is to orthogonalize the valence orbitals to the core orbitals; there are several ways to introduce the OVOs. One way is to construct the OVOs using

$$
\bar{\chi}_{v}^{\boldsymbol{k}}(\boldsymbol{r})=\chi_{v}^{\boldsymbol{k}}(\boldsymbol{r})-\sum_{c} \chi_{c}^{\boldsymbol{k}}(\boldsymbol{r}) S_{c v}^{\boldsymbol{k}} / S_{c c}^{\boldsymbol{k}},
$$


assuming that the core orbitals do not overlap each other. Another way is to construct the OVOs using

$$
\bar{\chi}_{v}^{\boldsymbol{k}}(\boldsymbol{r})=\sum_{p} \chi_{p}^{\boldsymbol{k}}(\boldsymbol{r}) S_{p v}^{\boldsymbol{k}-1}
$$

without any assumption. In the above equations, the indices $v$ and $c$ denote the valence and core orbitals, respectively, while the index $p$ denotes both classes of orbitals. Also, $\chi_{p}^{\boldsymbol{k}}(\boldsymbol{r})$ is the Bloch sum of the $p$-th atomic orbitals $\chi_{p}(\boldsymbol{r})$ associated with the wave vector $\boldsymbol{k}$ :

$$
\chi_{p}^{\boldsymbol{k}}(\boldsymbol{r})=\sum_{u} \exp \left(\mathrm{i} \boldsymbol{k} \cdot \boldsymbol{R}_{u}\right) \chi_{p}\left(\boldsymbol{r}-\boldsymbol{d}_{p}-\boldsymbol{R}_{u}\right),
$$

where $\boldsymbol{d}_{p}+\boldsymbol{R}_{u}$ represents the position of the center of the $p$-th atomic orbital in the $u$-th unit cell. Also $S_{p q}^{\boldsymbol{k}}$ is the overlap matrix defined as

$$
S_{p q}^{\boldsymbol{k}}=\int \chi_{p}^{\boldsymbol{k}}(\boldsymbol{r})^{*} \chi_{q}^{\boldsymbol{k}}(\boldsymbol{r}) \mathrm{d} \boldsymbol{r}
$$

and $S_{p q}^{\boldsymbol{k}-1}$ is its inverse matrix. In the present study, we use the OVOs constructed using eq. (2) throughout.

The next step of the OVOA is to solve the Kohn-Sham equations self-consistently in an approximate way; the valence states are obtained using the OVOs as the basis functions in the generalized eigenvalue problem while the core states are approximated by the Bloch sums given by eq. (3). The valence states are the solutions of the following eigenvalue equation of the Hamiltonian $H$ :

$$
H \psi_{n}^{\boldsymbol{k}}(\boldsymbol{r})=\varepsilon_{n}^{\boldsymbol{k}} \psi_{n}^{\boldsymbol{k}}(\boldsymbol{r}),
$$

and the corresponding charge density is calculated using

$$
\rho_{\mathrm{e}}^{\mathrm{v}}(\boldsymbol{r})=\frac{1}{N} \sum_{n \boldsymbol{k}} f_{n}^{\boldsymbol{k}} \psi_{n}^{\boldsymbol{k}}(\boldsymbol{r})^{*} \psi_{n}^{\boldsymbol{k}}(\boldsymbol{r})
$$

where $f_{n}^{\boldsymbol{k}}$ is the occupation number of the valence state $n \boldsymbol{k}$ and $N$ is the total number of $\boldsymbol{k}$. The valence wavefunction $\psi_{n}^{\boldsymbol{k}}(\boldsymbol{r})$ is then expanded using the OVOs as follows:

$$
\psi_{n}^{\boldsymbol{k}}(\boldsymbol{r})=\sum_{v} \bar{\chi}_{v}^{\boldsymbol{k}}(\boldsymbol{r}) \bar{C}_{v n}^{\boldsymbol{k}}
$$

This results in a usual generalized eigenvalue problem:

$$
\sum_{v^{\prime}} \bar{H}_{v v^{\prime}}^{\boldsymbol{k}} C_{v^{\prime} n}^{\boldsymbol{k}}=\varepsilon_{n}^{\boldsymbol{k}} \sum_{v^{\prime}} \bar{S}_{v v^{\prime}}^{\boldsymbol{k}} \bar{C}_{v^{\prime} n}^{\boldsymbol{k}}
$$

where

$$
\bar{H}_{v v^{\prime}}^{\boldsymbol{k}}=\int \bar{\chi}_{v}^{\boldsymbol{k}}(\boldsymbol{r})^{*} H \bar{\chi}_{v^{\prime}}^{\boldsymbol{k}}(\boldsymbol{r}) \mathrm{d} \boldsymbol{r}
$$

and

$$
\bar{S}_{v v^{\prime}}^{\boldsymbol{k}}=\int \bar{\chi}_{v}^{\boldsymbol{k}}(\boldsymbol{r})^{*} \bar{\chi}_{v^{\prime}}^{\boldsymbol{k}}(\boldsymbol{r}) \mathrm{d} \boldsymbol{r}
$$


On the other hand, the core states are assumed to be given by

$$
\psi_{c}^{\boldsymbol{k}}(\boldsymbol{r})=\chi_{c}^{\boldsymbol{k}}(\boldsymbol{r}) / \sqrt{S_{c c}^{\boldsymbol{k}}}
$$

which belongs to the eigenvalue

$$
\varepsilon_{c}^{\boldsymbol{k}}=\int \psi_{c}^{\boldsymbol{k}}(\boldsymbol{r})^{*} H \psi_{c}^{\boldsymbol{k}}(\boldsymbol{r}) \mathrm{d} \boldsymbol{r},
$$

and the corresponding charge density is calculated using

$$
\rho_{\mathrm{e}}^{\mathrm{c}}(\boldsymbol{r})=\frac{1}{N} \sum_{c \boldsymbol{k}} f_{c}^{\boldsymbol{k}} \psi_{c}^{\boldsymbol{k}}(\boldsymbol{r})^{*} \psi_{c}^{\boldsymbol{k}}(\boldsymbol{r}) .
$$

The total charge density is finally obtained as

$$
\rho_{\mathrm{e}}(\boldsymbol{r})=\rho_{\mathrm{e}}^{\mathrm{v}}(\boldsymbol{r})+\rho_{\mathrm{e}}^{\mathrm{c}}(\boldsymbol{r})
$$

and then the electrostatic and exchange-correlation potentials used in the next step of the self-consistent calculations are constructed using $\rho_{\mathrm{e}}(\boldsymbol{r})$. The extension of the above procedure to magnetic materials with spin polarizations is straightforward.

\section{Application to Au and InSb}

In our previous study, ${ }^{29)}$ we have reported the results of the FFLCAO and SFLCAO calculations on the structural and electronic properties of $\mathrm{Au}$ and $\mathrm{InSb}$ and the obtained results have been compared with those of experimental and other theoretical studies. Here, we compare the results obtained using the FFLCAO and SFLCAO calculations within the OVOA with the corresponding results obtained without the OVOA. The comparison with the results of other theoretical studies is, however, left out of the present paper because one can find it elsewhere. ${ }^{29)}$ We carried out the calculations employing the exchange-correlation energy functional within the local-spin-density approximation (LSDA) given by von Barth and Hedin. ${ }^{33)}$ We used 3096 and 2064 points per atom to perform three-dimensional numerical integration in real space for $\mathrm{Au}$ and $\mathrm{InSb}$, respectively. Also, we used $185 \boldsymbol{k}$ points generated using the good-lattice-point $\operatorname{method}^{34)}$ in the full Brillouin zone for Au while $16 \boldsymbol{k}$ points generated using the special-point $\operatorname{method}^{35)}$ in the full Brillouin zone for InSb. Furthermore, we performed the multipolar expansion of the electrostatic potential up to 8 .

We chose the basis functions so that they have enough variational flexibility. That is, we used not only the atomic orbitals of neutral atoms but also those of charged atoms. The atomic orbitals used for $\mathrm{Au}$ are the $1 s, 2 s, 2 p, 3 s, 3 p, 3 d, 4 s, 4 p, 4 d, 4 f, 5 s, 5 p, 5 d$, and $6 s$ atomic orbitals of neutral $\mathrm{Au}$ atoms, the $5 d$ and $6 s$ atomic orbitals of $\mathrm{Au}^{2+}$ atoms, and the $6 p$ atomic orbitals of $\mathrm{Au}^{+}$and $\mathrm{Au}^{3+}$ atoms; in the OVOA, the $1 s, 2 s, 2 p, 3 s, 3 p, 3 d, 4 s, 4 p, 4 d$, $4 f, 5 s$, and $5 p$ atomic orbitals are regarded as core orbitals, whereas the $5 d, 6 s$, and $6 p$ atomic orbitals are regarded as valence orbitals. The core and valence orbitals of Au are 68 (34) and 36 (18) in the FFLCAO (SFLCAO) calculations, respectively. The ratio of the number of the matrix elements to be calculated in the FFLCAO (SFLCAO) calculations without the OVOA 
to that in the FFLCAO (SFLCAO) calculations within the OVOA is found to be 8.3; this means that, for $\mathrm{Au}$, the FFLCAO (SFLCAO) calculations within the OVOA is about eight times faster than the FFLCAO (SFLCAO) calculations without the OVOA. Also, the atomic orbitals used for InSb are the $1 s, 2 s, 2 p, 3 s, 3 p, 3 d, 4 s, 4 p, 4 d, 5 s$, and $5 p$ atomic orbitals of neutral In and $\mathrm{Sb}$ atoms and the $5 s, 5 p$, and $5 d$ atomic orbitals of $\mathrm{In}^{2+}$ and $\mathrm{Sb}^{2+}$ atoms; in the OVOA, the $1 s, 2 s, 2 p, 3 s, 3 p, 3 d, 4 s$, and $4 p$ atomic orbitals of In and the $1 s, 2 s, 2 p$, $3 s, 3 p, 3 d, 4 s, 4 p$, and $4 d$ atomic orbitals of $\mathrm{Sb}$ are regarded as core orbitals, whereas the $4 d, 5 s, 5 p, 5 d$ atomic orbitals of In and the $5 s, 5 p, 5 d$ atomic orbitals of $\mathrm{Sb}$ are regarded as valence orbitals. We regard the $4 d$ atomic orbitals of In as valence orbitals because they are energetically not very deep. The core and valence orbitals of InSb are 82 (41) and 62 (31) in the FFLCAO (SFLCAO) calculations, respectively. The ratio of the number of matrix elements to be calculated in the FFLCAO (SFLCAO) calculations without the OVOA to that in the FFLCAO (SFLCAO) calculations within the OVOA is found to be 5.4; this means that, for InSb, the FFLCAO (SFLCAO) calculations within the OVOA is about five times faster than the FFLCAO (SFLCAO) calculations without the OVOA.

We now examine the accuracy of the OVOA in calculating the structural and electronic properties of Au. In Table I, we show the lattice constants and bulk moduli of Au obtained using the FFLCAO and SFLCAO calculations without the OVOA and those obtained using the FFLCAO and SFLCAO calculations within the OVOA; the experimental lattice constant and bulk modulus are also shown for comparison. The error in the lattice constant obtained using the FFLCAO calculations without the OVOA is $-0.4 \%$ and that obtained using the FFLCAO calculations within the OVOA is $-0.5 \%$. Also, the error in the lattice constant obtained using the SFLCAO calculations without the OVOA is less than $0.1 \%$ and that obtained using the SFLCAO calculations within the OVOA is $-0.1 \%$. Furthermore, the error in the bulk modulus obtained using the FFLCAO calculations without the OVOA is $+5 \%$ and that obtained using the FFLCAO calculations within the OVOA is $+4 \%$. Also, the error in the bulk modulus obtained using the SFLCAO calculations without the OVOA is $+9 \%$ and that obtained using the SFLCAO calculations within the OVOA is $+8 \%$. These results show that the differences between the results of the calculations without the OVOA and those of the calculations within the OVOA are sufficiently small for usual purposes.

In Fig. 1(a), the band structure of Au obtained using the FFLCAO calculations without the OVOA and that obtained using the FFLCAO calculations within the OVOA are shown and, in Fig. 1(b), the band structure of Au obtained using the SFLCAO calculations without the OVOA and that obtained using the SFLCAO calculations within the OVOA are shown; all the calculations were carried out using the experimental lattice constant. In these figures, the results of the calculations without the OVOA are drawn using red solid lines, while those of the calculations within the OVOA are drawn using blue dashed lines. It is found that 
Table I. Lattice constant (in $\AA$ ) and bulk modulus (in GPa) of Au. The errors in \% relative to the experimental results are shown in parenthesis.

\begin{tabular}{lcc}
\hline & Lattice constant & Bulk modulus \\
\hline Expt. $^{\text {a) }}$ & 4.078 & 173.2 \\
FFLCAO $^{\text {b) }}$ & $4.061(-0.4)$ & $182(+5)$ \\
FFLCAO within OVOA $\left.^{c}\right)$ & $4.056(-0.5)$ & $180(+4)$ \\
SFLCAO $^{\text {d }}$ & $4.076(0.0)$ & $189(+9)$ \\
${\text { SFLCAO within } \text { OVOA }^{\mathrm{e}}}^{\text {) }}$ & $4.072(-0.1)$ & $187(+8)$ \\
\hline
\end{tabular}

a) Reference 36 .

b) Fully relativistic full-potential LCAO calculations.

c) Fully relativistic full-potential LCAO calculations within orthogonalized valence orbital approximation.

d) Scalar relativistic full-potential LCAO calculations.

e) Scalar relativistic full-potential LCAO calculations within orthogonalized valence orbital approximation.

the results of the calculations without the OVOA and those of the calculations within the OVOA overlap almost perfectly. It should also be noted that the effect of spin-orbit coupling is successfully reproduced by the OVOA as seen in Fig. 1(a). Next, to examine the accuracy of the OVOA more quantitatively, we show the $d$-band widths obtained using the FFLCAO and SFLCAO calculations without the OVOA and those obtained using the FFLCAO and SFLCAO calculations within the OVOA in Table II. The difference between the result obtained using the FFLCAO calculations without the OVOA and that obtained using the FFLCAO calculations within the OVOA is $0.01 \mathrm{eV}$. The difference between the result obtained using the SFLCAO calculations without the OVOA and that obtained using the SFLCAO calculations within the OVOA is also $0.01 \mathrm{eV}$. This is the typical magnitude of the differences between the one-electron energies obtained using the calculations without the OVOA and those obtained using the calculations within the OVOA. The accuracy of the OVOA is thus found to be sufficiently good because one-electron energies usually differ by more than $0.1 \mathrm{eV}$ depending on the method employed for the calculations.

Finally, we show the calculated cohesive energies of $\mathrm{Au}$ as well as the experimental cohesive energy in Table III. The error in the cohesive energy obtained using the FFLCAO calculations without the OVOA is $+16 \%$ and that obtained using the FFLCAO calculations within the OVOA is also $+16 \%$. The difference between the calculated cohesive energies is $0.01 \mathrm{eV} /$ atom. Also, the error in the cohesive energy obtained using the SFLCAO calculations without the OVOA is $+11 \%$ and that obtained using the SFLCAO calculations within the OVOA is +10 


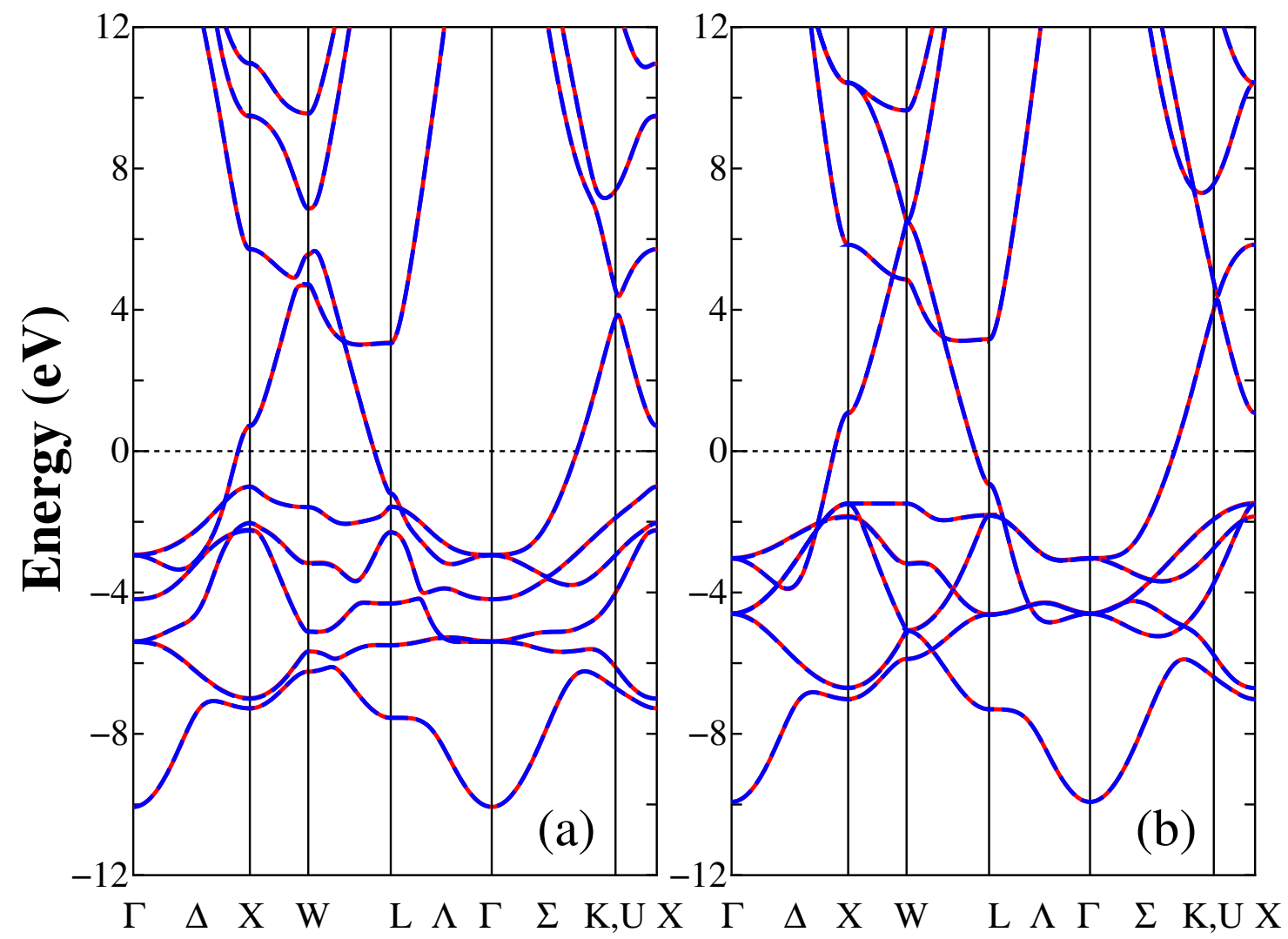

Fig. 1. Band structures of Au: (a)Results of the fully relativistic full-potential LCAO calculations (red solid lines) and those obtained within the orthogonalized valence orbital approximation (blue dashed lines). (b)Results of the scalar relativistic full-potential LCAO calculations (red solid lines) and those obtained within the orthogonalized valence orbital approximation (blue dashed lines). The red solid lines are traced almost perfectly by the blue dashed lines.

Table II. $\quad d$-band width (in eV) of Au.

\begin{tabular}{lc}
\hline & $d$-band width \\
\hline FFLCAO $^{\text {a) }}$ & 6.28 \\
FFLCAO within OVOA $^{\text {b) }}$ & 6.27 \\
SFLCAO $^{\text {c) }}$ & 5.55 \\
SFLCAO within OVOA $^{\text {d) }}$ & 5.54 \\
\hline
\end{tabular}

a) Fully relativistic full-potential LCAO calculations.

b) Fully relativistic full-potential LCAO calculations within orthogonalized valence orbital approximation.

c) Scalar relativistic full-potential LCAO calculations.

d) Scalar relativistic full-potential LCAO calculations within orthogonalized valence orbital approximation. 
Table III. Cohesive energy (in eV/atom) of $\mathrm{Au}$. The errors in \% relative to the experimental result are shown in parenthesis.

\begin{tabular}{cccccc}
\hline & Expt. $^{\text {a) }}$ & FFLCAO $^{\text {b) }}$ & $\begin{array}{c}\text { FFLCAO } \\
\text { within OVOA }\end{array}$ & SFLCAO $^{\text {d })}$ & $\begin{array}{c}\text { SFLCAO } \\
\text { within OVOA }\end{array}$ \\
\hline $\mathrm{Au}$ & 3.79 & $4.39(+16)$ & $4.38(+16)$ & $4.19(+11)$ & $4.17(+10)$ \\
\hline
\end{tabular}

a) Calculated from standard heat of formation at $298.15 \mathrm{~K}$.

b) Fully relativistic full-potential LCAO calculations.

c) Fully relativistic full-potential LCAO calculations within orthogonalized valence orbital approximation.

d) Scalar relativistic full-potential LCAO calculations.

e) Scalar relativistic full-potential LCAO calculations within orthogonalized valence orbital approximation.

\%. The difference between the calculated cohesive energies is $0.02 \mathrm{eV} /$ atom. Although the deviation of the calculated cohesive energies from the experimental one is noticeable due to the use of the LSDA, these results show that the OVOA is also a good approximation to the FFLCAO and SFLCAO calculations without the OVOA for obtaining cohesive energies.

We examine next the accuracy of the OVOA in calculating the structural and electronic properties of InSb. In Table IV, we show the lattice constants and bulk moduli of InSb obtained using the FFLCAO and SFLCAO calculations without the OVOA and those obtained using the FFLCAO and SFLCAO calculations within the OVOA; the experimental lattice constant and bulk modulus are also shown for comparison. The error in the lattice constant obtained using the FFLCAO calculations without the OVOA is $-0.2 \%$ and that obtained using the FFLCAO calculations within the OVOA is $+0.4 \%$. Also, the error in the lattice constant obtained using the SFLCAO calculations without the OVOA is $+0.1 \%$ and that obtained using the SFLCAO calculations within the OVOA is $+0.4 \%$. Furthermore, all the bulk moduli obtained using the present calculations are in agreement with each other as well as with the experimental bulk modulus within the fitting error of about $5 \%$.

In Fig. 2(a), the band structure of InSb obtained using the FFLCAO calculations without the OVOA and that obtained using the FFLCAO calculations within the OVOA are shown and, in Fig. 2(b), the band structure of InSb obtained using the SFLCAO calculations without the OVOA and that obtained using the SFLCAO calculations within the OVOA are shown; all the calculations were carried out using the experimental lattice constant. In these figures, the results of the calculations without the OVOA are drawn using red solid lines, while those of the calculations within the OVOA are drawn using blue dashed lines. It is found that the results of the calculations without the OVOA and those of the calculations within the OVOA overlap 
Table IV. Lattice constant (in $\AA$ ) and bulk modulus (in GPa) of InSb. The errors in \% relative to the experimental results are shown in parenthesis.

\begin{tabular}{lcc}
\hline & Lattice constant & Bulk modulus \\
\hline Expt. $^{\text {a) }}$ & 6.479 & 48.3 \\
FFLCAO $^{\text {b) }}$ & $6.463(-0.2)$ & $48(0)$ \\
FFLCAO within OVOA $^{c)}$ & $6.503(+0.4)$ & $48(0)$ \\
SFLCAO $^{\text {d }}$ & $6.488(+0.1)$ & $48(0)$ \\
SFLCAO within OVOA $^{\text {e) }}$ & $6.503(+0.4)$ & $48(0)$ \\
\hline
\end{tabular}

a) Reference 36 .

b) Fully relativistic full-potential LCAO calculations.

c) Fully relativistic full-potential LCAO calculations within orthogonalized valence orbital approximation.

d) Scalar relativistic full-potential LCAO calculations.

e) Scalar relativistic full-potential LCAO calculations within orthogonalized valence orbital approximation.

almost perfectly. It should also be noted that the effect of spin-orbit coupling is successfully reproduced by the OVOA as seen in Fig. 2(a). Next, to examine the accuracy of the OVOA more quantitatively, we show the one-electron energies at the $\Gamma, X$, and $L$ points obtained using the FFLCAO and SFLCAO calculations without the OVOA and those obtained using the FFLCAO and SFLCAO calculations within the OVOA in Table V. The difference between the results obtained using the FFLCAO calculations without the OVOA and those obtained using the FFLCAO calculations within the OVOA is at most $0.02 \mathrm{eV}$. Also, the difference between the results obtained using the SFLCAO calculations without the OVOA and those obtained using the SFLCAO calculations within the OVOA is at most $0.04 \mathrm{eV}$. The accuracy of the OVOA in calculating one-electron energies is again found to be sufficiently good as in the case of $\mathrm{Au}$.

Finally, we show the calculated cohesive energies of InSb as well as the experimental cohesive energy in Table VI. The error in the cohesive energy obtained using the FFLCAO calculations without the OVOA is $+12 \%$ and that obtained using the FFLCAO calculations within the OVOA is $+11 \%$. The difference between the calculated cohesive energies is 0.02 $\mathrm{eV} /$ atom. Also, the error in the cohesive energy obtained using the SFLCAO calculations without the OVOA is $+15 \%$ and that obtained using the SFLCAO calculations within the OVOA is $+14 \%$. The difference between the calculated cohesive energies is $0.02 \mathrm{eV} /$ atom. Although the deviation of the calculated cohesive energies from the experimental one is noticeable due to the use of the LSDA as in the case of Au, these results show that the OVOA 


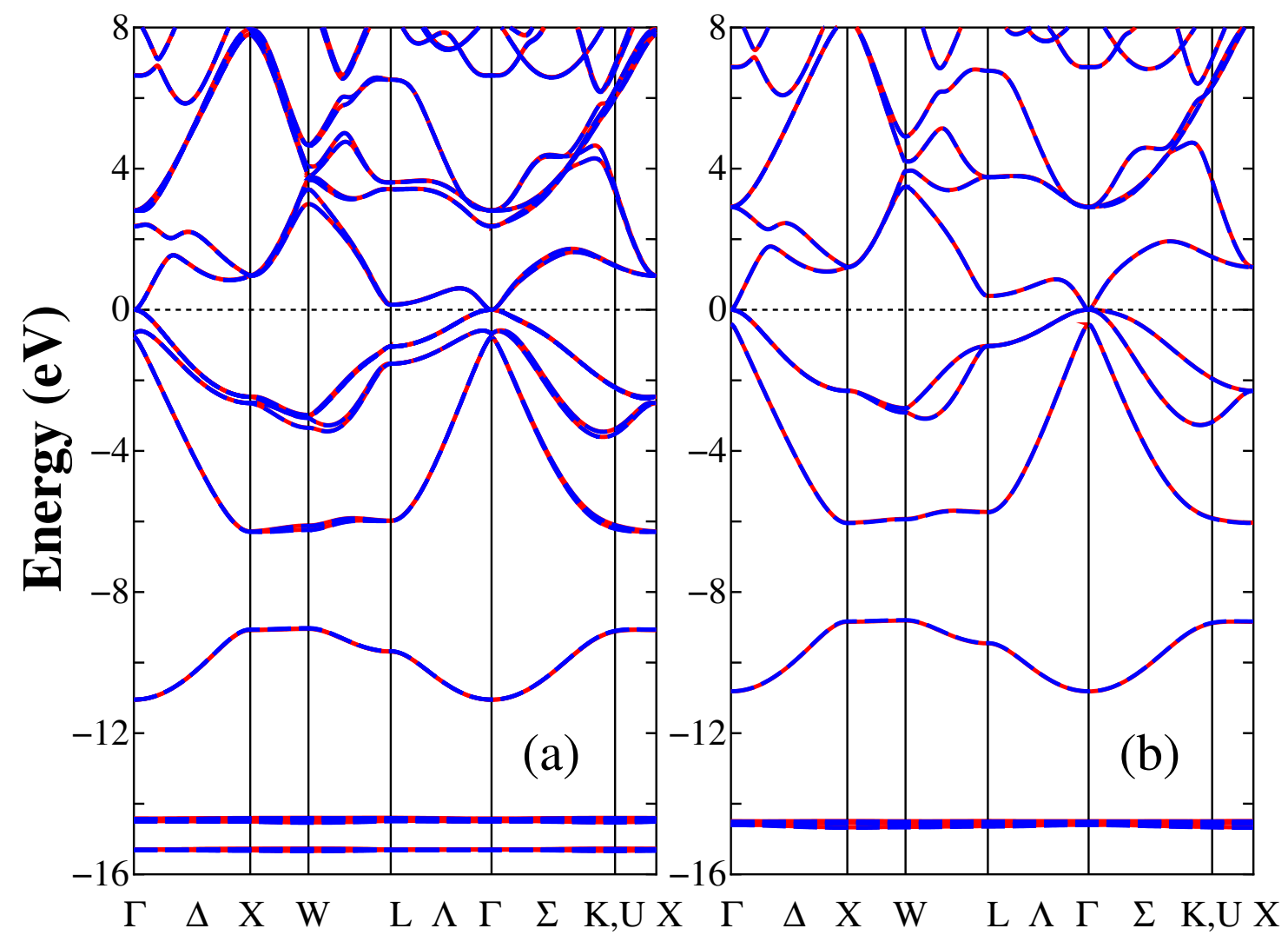

Fig. 2. Band structures of InSb: (a)Results of the fully relativistic full-potential LCAO calculations (red solid lines) and those obtained within the orthogonalized valence orbital approximation (blue dashed lines). (b)Results of the scalar relativistic full-potential LCAO calculations (red solid lines) and those obtained within the orthogonalized valence orbital approximation (blue dashed lines). The red solid lines are traced almost perfectly by the blue dashed lines.

is also a good approximation to the FFLCAO and SFLCAO calculations without the OVOA for obtaining cohesive energies.

\section{Electronic Structure of AmBi}

To show the applicability of our method to materials with very heavy atoms, we now study the electronic structure of AmBi using the FFLCAO and SFLCAO calculations. The results obtained within the OVOA are compared with those obtained without the OVOA. Furthermore, the present results are compared with the results of other theoretical studies reported previously. ${ }^{38,39)}$ This may be worthwhile because the results of the previous theoretical studies are in contradiction to each other; Petit et al. concluded that AmBi is a metal with a huge $f$ partial density of states at the Fermi level, ${ }^{38)}$ while Ghosh et al. concluded that $\mathrm{AmBi}$ is a narrow-gap semiconductor. ${ }^{39)}$

AmBi crystallizes in the rocksalt structure under ambient conditions; the observed lattice constant is $6.338 \AA$. The experimental studies have shown that AmBi is a material which 
Table V. One-electron energies (in $\mathrm{eV}$ ) of $\operatorname{InSb}$ at $\Gamma, X$, and $L$.

\begin{tabular}{|c|c|c|c|c|}
\hline & FFLCAO $^{a)}$ & $\begin{array}{c}\text { FFLCAO } \\
\text { within } \text { OVOA }^{\text {b) }}\end{array}$ & SFLCAO $^{\mathrm{c})}$ & $\begin{array}{c}\text { SFLCAO } \\
\text { within } \text { OVOA }^{\text {d) }}\end{array}$ \\
\hline \multirow[t]{2}{*}{$\Gamma_{15 c}$} & 2.82 & 2.81 & 2.95 & 2.91 \\
\hline & 2.38 & 2.36 & & \\
\hline$\Gamma_{1 c}$ & -0.65 & -0.67 & -0.38 & -0.41 \\
\hline \multirow[t]{2}{*}{$\Gamma_{15 v}$} & 0.00 & 0.00 & 0.00 & 0.00 \\
\hline & -0.78 & -0.77 & & \\
\hline$\Gamma_{1 v}$ & -11.06 & -11.05 & -10.81 & -10.81 \\
\hline$\Gamma_{12 v}$ & -14.42 & -14.44 & -14.44 & -14.51 \\
\hline \multirow[t]{2}{*}{$\Gamma_{25 v}$} & -14.48 & -14.50 & -14.54 & -14.61 \\
\hline & -15.29 & -15.31 & & \\
\hline$X_{3 c}$ & 0.99 & 0.97 & 1.25 & 1.21 \\
\hline$X_{1 c}$ & 0.98 & 0.96 & 1.24 & 1.20 \\
\hline \multirow[t]{2}{*}{$X_{5 v}$} & -2.46 & -2.47 & -2.28 & -2.30 \\
\hline & -2.64 & -2.65 & & \\
\hline$X_{3 v}$ & -6.29 & -6.29 & -6.01 & -6.05 \\
\hline$X_{1 v}$ & -9.08 & -9.06 & -8.85 & -8.83 \\
\hline \multirow[t]{2}{*}{$L_{3 c}$} & 3.62 & 3.61 & 3.80 & 3.76 \\
\hline & 3.42 & 3.41 & & \\
\hline$L_{1 c}$ & 0.15 & 0.14 & 0.42 & 0.39 \\
\hline \multirow[t]{2}{*}{$L_{3 v}$} & -1.04 & -1.04 & -1.02 & -1.03 \\
\hline & -1.53 & -1.53 & & \\
\hline$L_{1 v}$ & -5.98 & -5.99 & -5.71 & -5.74 \\
\hline$L_{1 v}$ & -9.69 & -9.68 & -9.46 & -9.45 \\
\hline
\end{tabular}

a) Fully relativistic full-potential LCAO calculations.

b) Fully relativistic full-potential LCAO calculations within orthogonalized valence orbital approximation.

c) Scalar relativistic full-potential LCAO calculations.

d) Scalar relativistic full-potential LCAO calculations within orthogonalized valence orbital approximation.

shows temperature-independent paramagnetism and possesses zero magnetic moment. ${ }^{40)}$ It is, however, still controversial whether the paramagnetic behavior is a Pauli paramagnetism or a Van Vleck paramagnetism; ${ }^{41)}$ the former corresponds to a metallic picture, while the latter corresponds to a narrow-gap semiconducting picture where the Am atoms are in the 
Table VI. Cohesive energy (in eV/atom) of InSb. The errors in \% relative to the experimental result are shown in parenthesis.

\begin{tabular}{cccccc}
\hline & Expt. $^{\text {a) }}$ & FFLCAO $^{\mathrm{b})}$ & $\begin{array}{c}\text { FFLCAO } \\
\text { within } \text { OVOA }^{\mathrm{c}}\end{array}$ & SFLCAO $^{\mathrm{d})}$ & $\begin{array}{c}\text { SFLCAO } \\
\text { within OVOA }\end{array}$ \\
\hline InSb & 2.80 & $3.13(+12)$ & $3.11(+11)$ & $3.21(+15)$ & $3.19(+14)$ \\
\hline
\end{tabular}

a) Calculated from standard heat of formation at $298.15 \mathrm{~K}$.

b) Fully relativistic full-potential LCAO calculations. ${ }^{37)}$

c) Fully relativistic full-potential LCAO calculations within orthogonalized valence orbital approximation.

d) Scalar relativistic full-potential LCAO calculations.

e) Scalar relativistic full-potential LCAO calculations within orthogonalized valence orbital approximation.

trivalent configuration, i.e., the $5 f^{6}$ configuration.

To compare the band structure obtained in the present study with that obtained by Ghosh et al., we employed the same exchange-correlation energy functional within the LSDA that they employed, i.e., the Vosko-Wilk-Nussair parametrization ${ }^{42)}$ of Ceperley and Alder's results. ${ }^{43)}$ We used 4644 points for the Am atom and 4128 points for the Bi atom to perform three-dimensional numerical integration in real space. Also, we used $32 \boldsymbol{k}$ points generated using the special-point method in the full Brillouin zone. Furthermore, we performed the multipolar expansion of the electrostatic potential up to 8 .

The atomic orbitals used for Am are the $1 s, 2 s, 2 p, 3 s, 3 p, 3 d, 4 s, 4 p, 4 d, 4 f, 5 s, 5 p, 5 d$, $5 f, 6 s, 6 p, 6 d$, and $7 s$ atomic orbitals of neutral Am atoms and the $5 f, 6 d$, and $7 s$ atomic orbitals of $\mathrm{Am}^{2+}$ atoms and $7 p$ atomic orbitals of $\mathrm{Am}^{+}$atoms, while the atomic orbitals used for $\mathrm{Bi}$ are the $1 s, 2 s, 2 p, 3 s, 3 p, 3 d, 4 s, 4 p, 4 d, 4 f, 5 s, 5 p, 5 d, 6 s$, and $6 p$ atomic orbitals of neutral $\mathrm{Bi}$ atoms and the $6 s, 6 p$, and $6 d$ atomic orbitals of $\mathrm{Bi}^{2+}$ atoms; in the OVOA, the $1 s$, $2 s, 2 p, 3 s, 3 p, 3 d, 4 s, 4 p, 4 d, 4 f, 5 s$, and $5 p$ atomic orbitals of Am and the $1 s, 2 s, 2 p, 3 s, 3 p$, $3 d, 4 s, 4 p, 4 d, 4 f, 5 s$, and $5 p$ atomic orbitals of $\mathrm{Bi}$ are regarded as core orbitals, while the $5 d$, $5 f, 6 s, 6 p, 6 d, 7 s$, and $7 p$ atomic orbitals of Am and the $5 d, 6 s, 6 p$, and $6 d$ atomic orbitals of $\mathrm{Bi}$ are regarded as valence orbitals. The core and valence orbitals of AmBi are 146 (73) and 102 (51) in the FFLCAO (SFLCAO) calculations, respectively. The ratio of the number of matrix elements to be calculated in the FFLCAO (SFLCAO) calculations without the OVOA to that in the FFLCAO (SFLCAO) calculations within the OVOA is found to be 5.9; this means that the FFLCAO (SFLCAO) calculations within the OVOA is about six times faster than the FFLCAO (SFLCAO) calculations without the OVOA.

In Fig. 3(a), the band structure of AmBi obtained using the FFLCAO calculations without 
the OVOA and that obtained using the FFLCAO calculations within the OVOA are shown and, in Fig. 3(b), the band structure of AmBi obtained using the SFLCAO calculations without the OVOA and that obtained using the SFLCAO calculations within the OVOA are shown; all the calculations were carried out using the experimental lattice constant. In these figures, the results of the calculations without the OVOA are drawn using red solid lines, while those of the calculations within the OVOA are drawn using blue dashed lines. It is found that the results of the calculations without the OVOA and those of the calculations within the OVOA overlap almost perfectly. As in $\mathrm{Au}$ and InSb, the effect of spin-orbit coupling is successfully reproduced by the OVOA as seen in Fig. 3(a). Next, to examine the accuracy of the OVOA more quantitatively, we show the energy gaps obtained using the FFLCAO and SFLCAO calculations without the OVOA and those obtained using the FFLCAO and SFLCAO calculations within the OVOA in Table VII. The difference between the result obtained using the FFLCAO calculations without the OVOA and that obtained using the FFLCAO calculations within the OVOA is less than $0.001 \mathrm{eV}$; the gap is indirect with the maximum of the valence band at the $\Gamma$ point, and the minimum of the conduction band at the $X$ point. The difference between the result obtained using the SFLCAO calculations without the OVOA and that obtained using the SFLCAO calculations within the OVOA is $0.014 \mathrm{eV}$; the gap is direct with both the maximum of the valence band and the minimum of the conduction band at the $X$ point. The typical magnitude of the difference between the one-electron energies obtained using the calculations without the OVOA and those obtained using the calculations within the OVOA is found to be about $0.01 \mathrm{eV}$. The accuracy of the OVOA is thus sufficiently good.

Our results obtained using the FFLCAO calculations are in agreement with those reported by Ghosh et al.; AmBi is a narrow-gap semiconductor with the indirect gap, where the maximum of the valence band is at the $\Gamma$ point, while the minimum of the conduction band is at the $X$ point. It is, however, found that there exist several discrepancies between our results and those reported by Ghosh et al.; for example, as shown in Table VII, the energy gap obtained using the FFLCAO calculations is about two times larger than that obtained by Ghosh et al. and a clear disagreement in the band structure is found in the vicinity of the $X$ point about $2 \mathrm{eV}$ below the Fermi level. Although these discrepancies may not be very serious, more detailed study is desirable to elucidate the electronic structure of AmBi accurately. This is also important to the investigation of the structural properties of $\mathrm{AmBi}$ because calculated structural properties are very sensitive to the accuracy of calculated electronic structures.

\section{Conclusions}

We have shown that the orthogonalized valence orbital approximation in the fully relativistic and scalar relativistic full-potential LCAO methods is useful for studying the structural and electronic properties of materials with heavy atoms. This approximation reduces the size 



$\Gamma \quad \Delta \quad \mathrm{X}$ W

$\mathrm{L} \Lambda \quad \Gamma \quad \Sigma \quad \mathrm{K}, \mathrm{U} X \quad \Gamma \quad \Delta \quad \mathrm{X} \quad \mathrm{W}$

$\mathrm{L} \Lambda \Gamma \Sigma \mathrm{K}, \mathrm{UX}$

Fig. 3. Band structures of AmBi: (a)Results of the fully relativistic full-potential LCAO calculations (red solid lines) and those obtained within the orthogonalized valence orbital approximation (blue dashed lines). (b)Results of the scalar relativistic full-potential LCAO calculations (red solid lines) and those obtained within the orthogonalized valence orbital approximation (blue dashed lines). The red solid lines are traced almost perfectly by the blue dashed lines.

of matrices in the generalized eigenvalue problem and thus reduces the computational cost of the calculations considerably. In particular, we have found that the effect of spin-orbit coupling is reproduced successfully in the fully relativistic full-potential LCAO calculations within the orthogonalized valence orbital approximation. 
Table VII. Energy gaps (in eV) of AmBi.

\begin{tabular}{lc}
\hline & Energy gap \\
\hline FFLCAO $^{\text {a) }}$ & 0.079 \\
FFLCAO within OVOA $^{\text {b) }}$ & 0.079 \\
SFLCAO $^{\text {c) }}$ & 0.117 \\
SFLCAO within OVOA $^{\text {d) }}$ & 0.131 \\
\hline Ref. 39 & 0.039 \\
\hline
\end{tabular}

a) Fully relativistic full-potential LCAO calculations.

b) Fully relativistic full-potential LCAO calculations within orthogonalized valence orbital approximation.

c) Scalar relativistic full-potential LCAO calculations.

d) Scalar relativistic full-potential LCAO calculations within orthogonalized valence orbital approximation.

e) Relativistic full-potential LMTO calculations including spin-orbit coupling carried out using a second variational procedure. 


\section{References}

1) M. Richter: Handbook of Magnetic Materials, ed. K. H. J. Buschow (Elsevier, Amsterdam, 2001), Vol. 13, p. 87.

2) A. O. Shorikov, A. V. Lukoyanov, M. A. Korotin, and V. I. Anisimov: Phys. Rev. B 72 (2005) 024458 .

3) T. L. Loucks: Phys. Rev. 139 (1965) A1333.

4) T. L. Loucks: Phys. Rev. 143 (1966) 506.

5) L. F. Mathesis: Phys. Rev. 151 (1966) 450.

6) D. D. Koelling: Phys. Rev. 188 (1969) 1049.

7) A. H. Macdonald, W. E. Pickett, and D. D. Koelling: J. Phys. C: Solid State Phys. 13 (1980) 2675.

8) H. Yamagami: Phys. Rev. B 61 (2000) 6246.

9) Y. Onodera and M. Okazaki: J. Phys. Soc. Jpn. 21 (1966) 1273.

10) S. Takada: Prog. Theor. Phys. 36 (1966) 224.

11) R. Feder, F. Rosicky, and B. Ackermann: Z. Phys. B 52 (1983) 31.

12) R. Feder, F. Rosicky, and B. Ackermann: Z. Phys. B 53 (1983) 244.

13) P. Strange, J. B. Staunton, and B. L. Gyorffy: J. Phys. C: Solid State Phys. 17 (1984) 3355.

14) H. Ebert, P. Strange, and B. L. Gyorffy: J. Phys. F 18 (1988) L135.

15) X. Wang, X.-G. Zhang. W. H. Butler, G. M. Stocks, and B. N. Harmon: Phys. Rev. B 46 (1992) 9352.

16) S. Bei der Kellen and A. J. Freeman: Phys. Rev. B 54 (1996) 11187.

17) N. E. Christensen: J. Phys. F 8 (1978) L51.

18) C. Godreche: J. Magn. Magn. Mater. 29 (1982) 262.

19) N. E. Christensen: Int. J. Quantum Chem. 25 (1984) 233.

20) H. Ebert: Phys. Rev. B 38 (1988) 9390.

21) H. Ebert, P. Strange, and B. L. Gyorffy: J. Appl. Phys. 63 (1988) 3052.

22) A. E. Krasovskii: Phys. Rev. B 60 (1999) 12788.

23) M. Richter and H. Eschrig: Solid State Commun. 72 (1989) 263.

24) X.-F. Zhong, Y.-N. Xu, and W. Y. Ching: Phys. Rev. B 41 (1990) 10545.

25) N. Mainkar, D. A. Browne, and J. Callaway: Phys. Rev. B 53 (1996) 3692.

26) J. C. Boettger: Phys. Rev. B 57 (1998) 8743.

27) S. Suzuki and K. Nakao: J. Phys. Soc. Jpn. 66 (1997) 3881.

28) S. Suzuki and K. Nakao: J. Phys. Soc. Jpn. 68 (1999) 1982.

29) S. Suzuki and K. Nakao: J. Phys. Soc. Jpn. 69 (2000) 532.

30) H. Yamagami and A. Hasegawa: J. Phys. Soc. Jpn. 59 (1990) 2426.

31) W. C. Herring: Phys. Rev. 57 (1940) 1169.

32) W. Y. Ching and Chun C. Lin.: Phys. Rev. B 12 (1975) 5536.

33) U. von Barth and L. Hedin: J. Phys. C: Solid State Phys. 5 (1972) 1629.

34) L.-K. Hua and Y. Wang: Applications of Number Theory to Numerical Analysis (Springer-Verlag, Berlin, 1981).

35) D. J. Chadi and M. L. Cohen: Phys. Rev. B 8 (1973) 5747.

36) Numerical Data and Functional Relationships in Science and Technology - Crystal and Solid State Physics, Vol. 17a of Landolt-Börnstein, ed. O. Madelung (Springer, Berlin, 1984). 
37) There was an error in Table XI of ref. 29; the calculated cohesive energy, $2.79 \mathrm{eV} /$ atom, of InSb obtained using the FFLCAO method should read as $3.13 \mathrm{eV} /$ atom as shown in Table VI of the present paper. The error occurred when the cohesive energy was calculated by subtracting the total energies of the isolated In and $\mathrm{Sb}$ atoms and thus none of the other results given in ref. 29 are affected.

38) L. Petit, A. Svane, W. M. Temmerman, and Z. Szotek: Phys. Rev. B 63 (2001) 165107.

39) D. B. Ghosh, S. K. De, P. M. Oppeneer, and M. S. S. Brooks: Phys. Rev. B 72 (2005) 115123.

40) B. Kanellakopulos, J. P. Charvillat, F. Maino, and W. Müller: Transplutonium, ed. W. Müller and R. Lindner (North-Holland, Amsterdam, 1975), p. 182.

41) T. Gouder, P. M. Oppeneer, F. Huber, F. Wastin, J. Rebizant: Phys. Rev. B 72(2005) 115122.

$42)$ S. H. Vosko, L. Wilk, and M. Nusair: Can. J. Phys. 58 (1980) 1200.

43) D. M. Ceperley and B. J. Alder: Phys. Rev. Lett. 45 (1980) 566. 